\title{
Validity and Reliability of Korean Version of Simplified Nutritional Appetite Questionnaire in Patients with Advanced Cancer: A Multicenter, Longitudinal Study
}

\author{
So Yeon $\mathrm{Oh}, \mathrm{MD}^{1}$ \\ Su-Jin Koh, MD, PhD² \\ Ji Yeon Baek, MD, PhD ${ }^{3}$ \\ Kyung A Kwon, MD, PhD 4 \\ Hei-Cheul Jeung, MD, PhD \\ Kyung Hee Lee, MD, $\mathrm{PhD}^{6}$ \\ Young-Woong Won, $\mathrm{MD}, \mathrm{PhD}^{7}$ \\ Hyun Jung Lee, MD
}

\begin{abstract}
${ }^{1}$ Division of Medical Oncology and Hematology, Department of Internal Medicine, Pusan National University Yangsan Hospital, Yangsan, ${ }^{2}$ Division of Hematology and Oncology, Department of Internal Medicine, Ulsan University Hospital, Ulsan University College of Medicine, Ulsan, ${ }^{3}$ Center for Colorectal Cancer, Research Institute and Hospital, National Cancer Center, Goyang, ${ }^{4}$ Division of Hematology and Oncology, Department of Internal Medicine, Dongnam Institute of Radiological and Medical Sciences, Busan, ${ }^{5}$ Division of Medical Oncology, Department of Internal Medicine, Gangnam Severance Hospital, Yonsei University College of Medicine, Seoul, ${ }^{6}$ Division of Hematology and Oncology, Department of Internal Medicine, Yeungnam University Hospital, Yeungnam University College of Medicine, Daegu, ${ }^{7}$ Division of Hematology and Oncology, Department of Internal Medicine, Hanyang University Guri Hospital, Guri, ${ }^{8}$ Division of Hematology and Oncology, Department of Internal Medicine, Dongguk University Ilsan Hospital, Goyang, Korea
\end{abstract}

Correspondence: Su-Jin Koh, MD, PhD Division of Hematology and Oncology, Department of Internal Medicine,

Ulsan University Hospital,

877 Bangeojinsunhwan-doro, Dong-gu,

Ulsan 44033, Korea

Tel: 82-52-250-8978

Fax: 82-52-250-7048

E-mail: sujinkoh@uuh.ulsan.kr

Received September 11, 2018

Accepted April 11, 2019

Published Online April 12, 2019

\section{Purpose}

Malnutrition and a loss of muscle mass are frequent in cancer patients and have a negative effect on clinical outcome. Nutrition risk screening aims to increase awareness and allow early recognition and treatment of cancer cachexia. Therefore, screenings should be brief, inexpensive, highly sensitive, and have good specificity. Simplified Nutritional Appetite Questionnaire (SNAQ) is a simple screening tool including four questions, and validated to predict weight loss within 6 months in community-dwelling adults and nursing home residents. Our study aimed to translate the SNAQ into Korean and to assess the validity and reliability of the translated screening tool in advanced cancer patients.

\section{Materials and Methods}

The SNAQ was translated into Korean according to linguistic validation. The internal consistency of the SNAQ was evaluated by Cronbach's alpha coefficient. Test-retest reliability was evaluated using the intraclass correlation coefficient. Concurrent validity was evaluated by measuring the Pearson's correlation coefficient between the SNAQ and Mini-Nutritional Assessment (MNA) and Patient-Generated Subjective Global Assessment (PG-SGA).

\section{Results}

In the 194 patients included in full analysis set, cancer stage was predominantly metastatic (98.5\%), the mean age was 60 years (range, 23 to 81 years), and the mean body mass index was $24 \mathrm{~kg} / \mathrm{m}^{2}$ (range, 15.6 to $39.6 \mathrm{~kg} / \mathrm{m}^{2}$ ). According to MNA score $\leq 11,57$ patients $(29.4 \%)$ were malnourished. The mean score ( \pm standard deviation) of the Korean version of the SNAQ was $13.8 \pm 2.5$ with a range of $6-19$. Cronbach's alpha coefficient was 0.737 , and intraclass correlation coefficient was 0.869 . The SNAQ was moderately correlated with MNA ( $r=0.404, p<0.001)$ and PG-SGA ( $r=-0.530, p<0.001)$. A significant weight loss of $>5 \%$ of the original body weight within 6 months occurred in 46 of the 186 patients $(24.7 \%)$. SNAQ score $\leq 14$ predicted $>5 \%$ weight loss with a sensitivity of $56.5 \%$ and a specificity of $44.3 \%$.

\section{Conclusion}

The Korean version of the SNAQ had high validity and reliability. SNAQ is useful for the screening tool for advanced cancer patients. The SNAQ had a limitation to predict impending weight loss in advanced cancer patients.
Key words

Nutritional assessment,

Simplified Nutritional Appetite Questionnaire, Neoplasms, Weight loss 


\section{Introduction}

Nutritional status affects clinical outcome in cancer patients. Impaired nutrition is associated with prolongation of admission, higher degree of treatment-related toxicity, poor quality of life, and a worse prognosis [1]. Patients at nutrition risk had a higher anticancer therapy-related adverse event $[2,3]$.

Screening of nutrition risk is important because it increases awareness and allows early recognition that enables early intervention [4]. Efficient screening tool should be brief, inexpensive, sensitive, and have a good specificity [5]. Several validated nutritional screening tool is available, e.g., Nutrition Risk Screening 2002 (NRS-2002) [6], Malnutrition Universal Screening Tool (MUST) [7], Malnutrition Screening Tool (MST) [8], Mini Nutritional Assessment Short Form (MNA-SF) [9]. However, these tools are too long or too complex to use in busy and crowded outpatient clinics. With the exception of MST, it is impossible for the patient to fill it up him (her)-self and needs the help of a skilled person.

The Simplified Nutritional Appetite Questionnaire (SNAQ) is short, simple appetite survey tool. It is originally developed for hospitalized patients of surgical and medical ward in the Netherlands to effectively screen malnourished patients [10]. It was also effective to predict weight loss in community adults and long-term care residents [11]. It consists of only four questions and could be filled up by patient alone without assistance. If SNAQ can be applied in cancer patients, nutritional screening can be done more easily and efficiently.

The purpose of the present study was to test the validity and reliability of the Korean version of SNAQ in advanced cancer patients. Also, we examined the correlation of SNAQ with existing assessment tools such as Mini-Nutritional Assessment (MNA) and Patient-Generated Subjective Global Assessment (PG-SGA).

\section{Materials and Methods}

\section{Study objectives and design}

Prior to this study, the SNAQ was translated into Korean according to linguistic validation. The primary endpoint of this study is the internal consistency of the Korean version of SNAQ evaluated by Cronbach's alpha coefficient. The secondary endpoints are (1) test-retest reliability that was evaluated using the intraclass correlation coefficient, (2) concurrent validity by measuring the Pearson's correlation coeffi- cient between the SNAQ and MNA and PG-SGA, (3) sensitivity and specificity of SNAQ according to weight loss. The study period of individual participants is 6 months (24 weeks) from the consent. The participants are requested to visit every 2 months from the first visit. The total period of this study is planned 1 year. In every visit, we performed history taking and physical exam including performance status and body weight. The SNAQ, MNA, and PG-SGA were checked in the baseline and last visit (fourth visit) which is 6 months from baseline. This study was conducted in eight institutions in South Korea. PG-SGA was conducted only in six institutions with available trained person to carry out the test.

\section{Sample size determination}

We searched the Cronbach's alpha coefficient of SNAQ from the previous studies to estimate sample size. Generally, the internal validity is evaluated as high when the Cronbach's alpha coefficient is 0.7 or more. However, SNAQ has only four items, and the coefficient is relatively low in the elderly and patients than the general population in prior studies. The Cronbach's alpha coefficient is between 0.51 and 0.61 in the elderly and chronically ill patients group [11-14]. In detail, Cronbach's alpha coefficient was 0.51 among residents of long-term care facilities and community-dwelling adults in St. Louis of the United States [11], and 0.55 in Japanese older adults people aged 65 years or more [12], 0.58 among patients in out-patients or in-patients department of Malaysian general hospital [13], and 0.61 in Brazilians of the cardiopulmonary and metabolic rehabilitation program [14]. Therefore, we expected Cronbach's alpha coefficient under the null hypothesis of 0.50 and alternative hypothesis of 0.65 among recurrent or metastatic cancer patients. We decided the significance level was 0.05 and the power was $80 \%$, and the number of required subjects was 163 . Considering the drop-out rate of $10 \%$ to last visit, a total of 182 people were required [15]. To calculate the sample size, we used the following formula:

$$
\mathrm{n}=\frac{\left\{\frac{2 \mathrm{k}}{(\mathrm{k}-1)}\right\}\left(z_{\alpha / 2}+z_{\beta}\right)^{2}}{\operatorname{In}\left\{\frac{\left(1-p_{0}\right)}{\left(1-p_{1}\right)}\right\}^{2}}+2
$$

where, $\mathrm{k}=4, \alpha=0.05, \beta=0.2, p_{0}=0.5, p_{1}=0.65$.

\section{Patient}

This study includes patients who (1) aged 20 years old or more, (2) diagnosed with recurrent or metastatic cancer which is not curable, (3) have life expectancy as six months 
or more, (4) can read and understand document written in Korean, and (5) signed informed consent. Patients were excluded if he/she (1) cannot read and write survey (i.e., blindness, severe weakness), (2) has current ongoing mental illness (i.e., major depression, bipolar disorder) or cognitive dysfunction, (3) has intracranial tumor including brain metastasis, (4) have received major surgery within prior 8 weeks or scheduled major surgery within the study periods, (5) need enteral feeding or parenteral nutrition, or therapeutically restricted diet, (6) have grade 3 or 4 nausea or vomiting induced by chemotherapy or radiotherapy, (7) cannot tolerate oral nutrition due to intestinal dysfunction like peritoneal seeding or bowel obstruction, (8) participating another clinical trial or have plan to participate during the study period, (9) is not appropriate to participate in the study under the decision of the investigator.

\section{Statistical analysis}

The internal consistency of the SNAQ was evaluated by Cronbach's alpha coefficient. The primary endpoint was identified based on the first SNAQ created, and the SNAQ created during the second and last visit was analyzed as an auxiliary value. In factor analysis, Varimax rotation method was applied. Test-retest reliability was evaluated using the intraclass correlation coefficient (ICC). ICC was calculated from SNAQ, which was surveyed twice at 2- to 8-hour interval at baseline visit. For calculation of ICC, only the subjects who completed SNAQ twice at the baseline visit were included. Concurrent validity was evaluated by measuring the Pearson's correlation coefficient between the SNAQ and MNA or PG-SGA. The correlation coefficient was measured at baseline and last visit. The continuous variables were presented as descriptive statistics (mean, standard deviation) and categorical variables were number (\%), A 2-tailed p-value 0.05 was considered significant. All statistical analyses are done by SAS ver. 9.4 (SAS Institute Inc., Cary, NC).

\section{Ethical statement}

The protocol was approved by the institutional review boards of each participating hospital and performed in accordance with the principles of the Declaration of Helsinki and the Good Clinical Practice Guidelines defined by the International Conference on Harmonization. All patients provided written, informed consent before enrollment.
Table 1. Baseline characteristics of all participants

\begin{tabular}{lc}
\hline Variable & Total $(\mathbf{n}=194)$ \\
\hline Age $(\mathbf{y r})$ & $60.0 \pm 10.3$ \\
Sex & \\
Male & $107(55.2)$ \\
\hline Female & $87(44.8)$ \\
Diagnosis & \\
\hline Breast cancer & $33(17.0)$ \\
\hline Colon cancer & $28(14.4)$ \\
\hline Rectal cancer & $19(9.8)$ \\
\hline Cholangiocarcinoma & $13(6.7)$ \\
\hline Gastric cancer & $12(6.2)$ \\
\hline Lung neoplasm malignant & $11(5.7)$ \\
\hline Others & $78(40.2)$ \\
\hline Stage & \\
\hline I & 0 \\
\hline II & $3(1.5)$ \\
\hline III & $7(3.6)$ \\
\hline IV & $162(83.5)$ \\
\hline Others & $2(1.0)$ \\
\hline Missing & $20(10.3)$ \\
\hline Metastatic disease & \\
\hline Yes & $191(98.5)$ \\
\hline No & $3(1.6)$ \\
\hline ECOG PS & $20(10.3)$ \\
\hline 0 & $154(79.4)$ \\
\hline 1 & $19(9.8)$ \\
\hline 2 & $1(0.5)$ \\
\hline 3 & 0 \\
\hline 4 & $162.1 \pm 8.3$ \\
\hline Height $(\mathbf{c m})$ & $63.1 \pm 10.8$ \\
\hline Weight $(\mathbf{k g})$ & $24.0 \pm 3.7$ \\
\hline BMI $\left(\mathbf{k g} / \mathbf{m}^{2}\right)$ & $191(98.5)$ \\
\hline Have a meal & $3(1.5)$ \\
\hline Without help & \\
\hline With help & \\
\hline & \\
\hline
\end{tabular}

Values are presented as mean \pm standard deviation or number (\%). ECOG PS, Eastern Cooperative Oncology Group performance status; BMI, body mass index.

\section{Results}

\section{Patients' characteristics}

The number of total participants was 194 at baseline. Among them, 155 patients $(79.9 \%)$ completed the study to the end. The reasons of drop-out were the withdrawal of consent $(n=16)$, death $(n=14)$, failure to follow up $(n=5)$, violation of eligibility criteria $(n=1)$, and investigator's decision $(n=3)$. 
Table 2. The result of SNAQ and the internal consistency

\begin{tabular}{|c|c|c|c|}
\hline & $\begin{array}{l}\text { Visit } 1 \text { initial } \\
\quad(n=194)\end{array}$ & $\begin{array}{l}\text { Visit 12nd } \\
(\mathrm{n}=194)\end{array}$ & $\begin{array}{l}\text { Visit } 4 \\
(n=156)\end{array}$ \\
\hline \multicolumn{4}{|l|}{ SNAQ score } \\
\hline Mean \pm SD & $13.8 \pm 2.5$ & $13.9 \pm 2.5$ & $13.6 \pm 2.4$ \\
\hline Median & 14.0 & 14.0 & 14.0 \\
\hline Min-max & $6-19$ & $4-19$ & $4-19$ \\
\hline $0-14, \mathrm{n}(\%)$ & $111(57.2)$ & $114(58.8)$ & $101(64.7)$ \\
\hline $15-20, \mathrm{n}(\%)$ & $83(42.8)$ & $80(41.2)$ & $55(35.3)$ \\
\hline \multicolumn{4}{|l|}{ Internal consistency } \\
\hline Cronbach's alpha coefficient $(p)$ & $0.737^{\mathrm{a})}$ & 0.747 & 0.652 \\
\hline
\end{tabular}

SNAQ, Simplified Nutritional Appetite Questionnaire; SD, standard deviation. ${ }^{\text {a) }}$ A test for the hypothesis $H_{0}: p=p_{0}=0.5$ revealed $\mathrm{p}<0.05$.

Table 3. Factor analyses by Varimax rotation method to evaluate the similarity between items

\begin{tabular}{|c|c|c|c|c|c|}
\hline \multirow{2}{*}{ Factor } & \multicolumn{4}{|c|}{ Factorial loads $^{\text {a) }}$} & \multirow{2}{*}{$\begin{array}{l}\text { Variance explained } \\
\text { by factor }\end{array}$} \\
\hline & Item 1 & Item 2 & Item 3 & Item 4 & \\
\hline Factor 1 & 0.380 & 0.144 & 0.934 & 0.101 & 1.047 \\
\hline Factor 2 & 0.256 & 0.962 & 0.150 & 0.117 & 1.027 \\
\hline Factor 3 & 0.205 & 0.121 & 0.108 & 0.976 & 1.021 \\
\hline Factor 4 & 0.865 & 0.198 & 0.306 & 0.154 & 0.906 \\
\hline
\end{tabular}

${ }^{a}$ Each item is as follows: item 1, my appetite is; item 2, when I eat (satiety); item 3, food tastes; item 4, normally I eat (amount).

The baseline characteristics of participants are presented in Table 1. Majority of patients $(n=153,78.9 \%)$ had comorbidities besides cancer. Concomitant diseases are vascular disorders including hypertension $(n=66)$, metabolic disease including diabetes $(n=53)$, musculoskeletal disorders $(n=28)$, nervous system disorder $(n=26)$, infections $(n=24)$, gastrointestinal disorders $(n=23)$, respiratory disorders $(n=18)$, psychiatric disorders $(n=14)$, and others.

\section{Internal consistency of the SNAQ Korean version}

The details of SNAQ score per visit are presented in Table 2. The Cronbach's alpha coefficient was between 0.75 and 0.65 , indicating that the internal consistency is acceptable ( $\mathrm{p}<$ 0.05). In the factor analysis using Varimax rotation method, the variance explained by the factors ranged from 0.9056 to 1.0467. Therefore, it is evaluated that there is no overlapping concept item among the current four items of SNAQ (Table 3).

\section{Test-retest reliability by ICC}

The test-retest reliability measured by ICC of SNAQ from two measurements of visit 1 are presented in Table 4 . The
Table 4. Test-retest reliability of SNAQ from two measurements at baseline visit

\begin{tabular}{ll} 
& ICC \\
Item 1. My appetite is & 0.885 \\
Item 2. When I eat (satiety) & 0.846 \\
Item 3. Food tastes & 0.843 \\
Item 4. Normally I eat (amount) & 0.902 \\
Mean & 0.869 \\
\hline
\end{tabular}

SNAQ, Simplified Nutritional Appetite Questionnaire; ICC, intraclass correlation coefficient.

ICC values are bigger than 0.8 , showing excellent agreement.

\section{MNA, PG-SGA, and concurrent validity of SNAQ}

The mean MNA score were $12.0 \pm 2.3$ and $12.1 \pm 2.1$ and total 61 patients $(31.4 \%)$ and 46 patients $(29.5 \%)$ were found to be at risk of malnutrition (MNA score $<12$ ) at visit 1 and visit 4 , respectively. Among them, only nine and five patients had malnutrition by definition of malnutrition indicator score 
Table 5. Pearson's correlation coefficient between SNAQ and MNA, or PG-SGA at visit 1 and visit 4

\begin{tabular}{|c|c|c|}
\hline Visit & $\begin{array}{c}\text { Visit } 1 \\
\text { SNAQ (initial) }\end{array}$ & $\begin{array}{l}\text { Visit } 4 \\
\text { SNAQ }\end{array}$ \\
\hline \multicolumn{3}{|l|}{ Visit 1} \\
\hline MNA & 194 & - \\
\hline No. & 0.404 & \\
\hline Correlation coefficient & $<0.001$ & \\
\hline \multicolumn{3}{|l|}{$\mathrm{p}$-value } \\
\hline \multicolumn{3}{|l|}{ PG-SGA } \\
\hline No. & 152 & - \\
\hline Correlation coefficient & -0.530 & \\
\hline $\mathrm{p}$-value & $<0.001$ & \\
\hline \multicolumn{3}{|l|}{ Visit 4} \\
\hline \multicolumn{3}{|l|}{ MNA } \\
\hline No. & - & 156 \\
\hline Correlation coefficient & & 0.485 \\
\hline $\mathrm{p}$-value & & $<0.001$ \\
\hline \multicolumn{3}{|l|}{ PG-SGA } \\
\hline No. & - & 123 \\
\hline Correlation coefficient & & -0.590 \\
\hline p-value & & $<0.001$ \\
\hline
\end{tabular}

SNAQ, Simplified Nutritional Appetite Questionnaire; MNA, Mini-Nutritional Assessment; PG-SGA, Patient-Generated Subjective Global Assessment.

Table 6. Subgroup analyses of sensitivity and specificity of SNAQ in prediction of 5\% or more weight loss within 6 months

\begin{tabular}{|c|c|c|c|c|}
\hline & \multirow{2}{*}{ No. } & \multicolumn{3}{|c|}{ Weight loss more than $5 \%$} \\
\hline & & No. & Sensitivity $(\%)$ & Specificity $(\%)$ \\
\hline Total & 186 & 46 & 56.5 & 44.3 \\
\hline \multicolumn{5}{|l|}{ Sex } \\
\hline Male & 104 & 32 & 50.0 & 61.1 \\
\hline Female & 82 & 14 & 71.4 & 26.5 \\
\hline \multicolumn{5}{|l|}{ Age (yr) } \\
\hline$<65$ & 118 & 24 & 37.5 & 41.5 \\
\hline$\geq 65$ & 68 & 22 & 77.3 & 50.0 \\
\hline \multicolumn{5}{|c|}{ Gastrointestinal symptom } \\
\hline Present & 121 & 28 & 60.7 & 44.1 \\
\hline None & 65 & 18 & 50.0 & 44.7 \\
\hline \multicolumn{5}{|l|}{ Chemotherapy } \\
\hline Ongoing & 153 & 34 & 52.9 & 43.7 \\
\hline Not ongoing & 33 & 12 & 66.7 & 47.6 \\
\hline
\end{tabular}

SNAQ, Simplified Nutritional Appetite Questionnaire.

under 17. Meanwhile, the PG-SGA was measured only in institutions where trained person is available to carry out the assessment. Six out of eight institutions conducted PG-SGA in 152 patients. Among them, 34 patients (22.4\%) at visit 1 and 32 patients $(26.0 \%)$ at visit 4 were suspected moderate malnutrition in PG-SGA. Four patients are severely malnourished per each visit. Finally, MNA and PG-SGA were analyzed with SNAQ to evaluate concurrent validity. The Pearson's correlation coefficient between SNAQ and MNA, or PG-SGA are presented in Table 5. SNAQ have clear posi- 
tive correlation with MNA at both visit 1 and visit 4. On the contrary, SNAQ have clear negative correlation with PGSGA at both visits.

\section{Sensitivity and specificity of SNAQ in prediction of weight loss}

A significant weight loss of $>5 \%$ of the original body weight within 6 months occurred in 46 of the 186 patients $(24.7 \%)$. SNAQ score $\leq 14$ predicted $>5 \%$ weight loss with a sensitivity of $56.5 \%$ and a specificity of $44.3 \%$. In subgroup analyses, the sensitivity and specificity of male, older $(\geq 65$ years) patients, patients with gastrointestinal symptom, and patients who were not receiving chemotherapy were better than female, younger ones, patients without gastrointestinal symptom, and who was receiving chemotherapy (Table 6). In these subgroups, however, sensitivity exceeded $60 \%$, but the specificity did not exceed $50 \%$ in most cases. Therefore, no useful results were found in the prediction of weight loss by SNAQ.

\section{Discussion}

In this study, we investigate the significance of the SNAQ as a quick screening tool for nutritional status of advanced cancer patients. In my knowledge, this is the first nutritional screening tool for cancer patients which was validated in Korea. The Cronbach's alpha coefficient, the primary endpoint, was above 0.7 at baseline visit. A commonly accepted rule for describing internal consistency using Cronbach's alpha is as follows: if $<0.6$, poor; between 0.6 and 0.7 , questionable; between 0.7 and 0.8 , acceptable; between 0.8 and 0.9 , good; above 0.9 , excellent [16]. Thus, we found that the internal consistency of Korean version SNAQ is acceptable. In the factor analysis, that there is no overlapping item in the current four items of SNAQ. Additionally, the test-retest reliability is excellent and SNAQ has clear correlation with existing nutritional assessment tools such as MNA and PG-SGA. As a result, we found that SNAQ is sufficiently reliable and valid tool in nutritional screening of cancer patients.

Malnutrition deteriorate the clinical outcome and increase financial burden in cancer patients $[3,5]$. Therefore, importance of early nutritional screening in cancer patients is increasing. In one study, higher degree of nutritional screening is associated with lower prevalence of malnutrition over time period of 4 years [4]. In other words, nutrition screening itself prevented malnutrition. Quick nutritional screening should be done to identify patients at risk of malnutrition to proceed to further nutritional assessment.

For nutritional screening, several validated tools are commonly used. NRS-2002 is extensively validated in hospitalized patients to predict morbidity, complication, and length of admission [6]. MUST is validated in similar in-patient setting [17]. Though these two screening tools showed high reproducibility and good correlation with clinical outcome, they cannot be filled up by the patient alone and information about disease severity is required to be completed. MNA is developed to screen elderly people but should be filled up by trained person because its items include calf circumference and psychological problems [18]. MST consists of only two items and correlates with length of admission in in-patient setting [8]. But, MST is not validated for out-patients or cancer patients. These tools (NRN-2002, MUST, and MNA) have validated role in selecting nutritional risk groups. However, it is hard to implement screening using these tools in busy and crowded outpatient clinic due to constraints of time and space.

SNAQ is developed for screening of nutritional risk in hospitalized patients. The original population is inpatients of surgical and medical wards including about $20 \%$ of cancer patients. Screening with SNAQ was associated with shorter hospital stay [10]. SNAQ consists of only four questions about dietary habits and appetite. The advantage of SNAQ over other tools is that it can be completed by the subject alone without the help of a trained person because it is short and easy. We expect this tool to be applicable to all cancer patients who will be diagnosed as advanced cancer even in a busy medical field such as outpatient department or day hospital.

However, the sensitivity of SNAQ to weight loss exceeding $5 \%$ was $56.5 \%$ and specificity was $44.3 \%$ that showed a score of almost no discrimination power of $50 \%$. In the receiver operating characteristic (ROC) analysis, in the same manner, the area under the curve of the ROC curve was 0.516 (95\% confidence interval, 0.407 to 0.625 ) that means there is no significance. The results of the ROC analysis for the prediction of over 10\% reduction in body weight were also not significant. In the subgroup analyses, it seemed that SNAQ has better performance in male, the older patients, those with gastrointestinal symptom, and who was not receiving chemotherapy in compared to their counterpart group. However, there was no subgroup that had acceptable sensitivity and specificity of SNAQ. Most advanced cancer patients have multiple factors affecting appetite or dietary habits that concern body weight regardless of pattern of food intake such as change of taste and gastrointestinal function, reduced physical activity, metabolic derangement [5]. Accordingly, SNAQ may be less sensitive or specific in compared to community-dwelling elderlies who were original subject population of initial study of it [11]. 
In MNA, around 30\% of patients were at risk of malnutrition. Similarly, about $25 \%$ of patients were suspected as moderate malnutrition by PG-SGA. On the other hand, the overt (or severe) malnutrition (4 to 9 patients) were rare by both MNA and PG-SGA. The prevalence of malnutrition cancer patients has been reported to range from around $20 \%$ to more than $70 \%$ worldwide according to region or countries [2,3,19-21]. The prevalence of malnutrition in this study is quite low even when compared to the previous study of National Cancer Center of Korea which described the prevalence of malnutrition is $61 \%$ among hospitalized cancer patients [22]. The low prevalence of our study is probably because the selection and exclusion criteria enabled the participation of only patients who can eat food with an expected survival time of at least 6 months.

Our study has several limitations. First, the SNAQ have low sensitivity and specificity in predicting weight loss though its correlation with previously proven tool such as MNA and PG-SGA is good. Thus, it renders concern that SNAQ may not be predictive of weight loss within future 6 months in cancer patients. At least, however, we can assume that SNAQ is related to the nutritional status of the patient at that time. Therefore, it is a little comfort to us that the role of SNAQ as a nutritional screening tool has not been compromised. Second, the drop-out rate is unexpectedly high. The originally planned number of required subjects was 182 considering the drop-out rate of $10 \%$. In practice, we enrolled 194 patients but only 155 patients complied with to the end of the study. Death was the cause of drop-out in 14 patients that is not beyond expectations. However, the number of patients withdrawing consent was higher than originally anticipated $(\mathrm{n}=16)$. No data were collected on the reasons why the patient withdrew consent. Although the planned number of patients could not be met, fortunately, the primary endpoint was met. Lastly, SNAQ was validated in patients with advanced, incurable cancer but not imminent death in our study. The SNAQ does not provide any information about a nutritional screening in other situation of cancer patients such as postoperative periods, neo- or adjuvant treatment setting, or terminal state.

In conclusion, the Korean version of the SNAQ had acceptable internal consistency, high validity, and reliability. SNAQ is useful tool for the nutritional screening of advanced cancer patients. SNAQ had a limitation to predict weight loss of near future in advanced cancer patients. However, SNAQ was well correlated with current nutritional status of cancer patients which was determined by previously proven assessment tools. In a busy clinical field, a simple and easy search tool like SNAQ is expected to help early detection of malnutrition in cancer patients.

\section{Conflicts of Interest}

This study was sponsored by Boryung Pharmaceutical Co., Ltd. The sponsor was involved only in the initial phase of the study design. All other activities, including final study design, subject enrollment, study conduct, data acquisition and analysis, presentation of the data, and writing manuscript were carried out by the authors and/or those whom they delegated under their supervision.

\section{References}

1. Caccialanza R, Pedrazzoli P, Cereda E, Gavazzi C, Pinto C, Paccagnella A, et al. Nutritional support in cancer patients: a position paper from the Italian Society of Medical Oncology (AIOM) and the Italian Society of Artificial Nutrition and Metabolism (SINPE). J Cancer. 2016;7:131-5.

2. Pan H, Cai S, Ji J, Jiang Z, Liang H, Lin F, et al. The impact of nutritional status, nutritional risk, and nutritional treatment on clinical outcome of 2248 hospitalized cancer patients: a multi-center, prospective cohort study in Chinese teaching hospitals. Nutr Cancer. 2013;65:62-70.

3. Arends J, Baracos V, Bertz H, Bozzetti F, Calder PC, Deutz NE, et al. ESPEN expert group recommendations for action against cancer-related malnutrition. Clin Nutr. 2017;36:1187-96.

4. Meijers JM, Tan F, Schols JM, Halfens RJ. Nutritional care: do process and structure indicators influence malnutrition prevalence over time? Clin Nutr. 2014;33:459-65.

5. Arends J, Bachmann P, Baracos V, Barthelemy N, Bertz H,
Bozzetti F, et al. ESPEN guidelines on nutrition in cancer patients. Clin Nutr. 2017;36:11-48.

6. Kondrup J, Rasmussen HH, Hamberg O, Stanga Z; Ad Hoc ESPEN Working Group. Nutritional risk screening (NRS 2002): a new method based on an analysis of controlled clinical trials. Clin Nutr. 2003;22:321-36.

7. Stratton RJ, Hackston A, Longmore D, Dixon R, Price S, Stroud $\mathrm{M}$, et al. Malnutrition in hospital outpatients and inpatients: prevalence, concurrent validity and ease of use of the 'malnutrition universal screening tool' ('MUST') for adults. Br J Nutr. 2004;92:799-808.

8. Ferguson M, Capra S, Bauer J, Banks M. Development of a valid and reliable malnutrition screening tool for adult acute hospital patients. Nutrition. 1999;15:458-64.

9. Rubenstein LZ, Harker JO, Salva A, Guigoz Y, Vellas B. Screening for undernutrition in geriatric practice: developing the short-form mini-nutritional assessment (MNA-SF). J 
Gerontol A Biol Sci Med Sci. 2001;56:M366-72.

10. Kruizenga HM, Van Tulder MW, Seidell JC, Thijs A, Ader HJ, Van Bokhorst-de van der Schueren MA. Effectiveness and cost-effectiveness of early screening and treatment of malnourished patients. Am J Clin Nutr. 2005;82:1082-9.

11. Wilson MM, Thomas DR, Rubenstein LZ, Chibnall JT, Anderson S, Baxi A, et al. Appetite assessment: simple appetite questionnaire predicts weight loss in community-dwelling adults and nursing home residents. Am J Clin Nutr. 2005;82:1074-81.

12. Nakatsu N, Sawa R, Misu S, Ueda Y, Ono R. Reliability and validity of the Japanese version of the simplified nutritional appetite questionnaire in community-dwelling older adults. Geriatr Gerontol Int. 2015;15:1264-9.

13. Hanisah R, Suzana S, Lee FS. Validation of screening tools to assess appetite among geriatric patients. J Nutr Health Aging. 2012;16:660-5.

14. Sties SW, Gonzales AI, da Silveira Viana M, Brandt R, Bertin RL, Goldfeder R, et al. Simplified Nutritional Appetite Questionnaire (SNAQ) for cardiopulmonary and metabolic rehabilitation program. Rev Bras Med Esporte. 2012;18:313-7.

15. Bonett DG. Sample size requirements for testing and estimating coefficient alpha. J Educ Behav Stat. 2002;27:335-40.

16. George D, Mallery P. SPSS for Windows step by step: a simple guide and reference, fourth edition (11.0 update). Boston, MA:
Allyn \& Bacon; 2003.

17. King CL, Elia M, Stroud MA, Stratton RJ. The predictive validity of the malnutrition universal screening tool (MUST) with regard to mortality and length of stay in elderly inpatients. Clin Nutr. 2003;22(Suppl 1):S4.

18. Guigoz Y. The Mini Nutritional Assessment (MNA) review of the literature: what does it tell us? J Nutr Health Aging. 2006; 10:466-85.

19. Planas M, Alvarez-Hernandez J, Leon-Sanz M, Celaya-Perez S, Araujo K, Garcia de Lorenzo A, et al. Prevalence of hospital malnutrition in cancer patients: a sub-analysis of the PREDyCES(R) study. Support Care Cancer. 2016;24:429-35.

20. Pressoir M, Desne S, Berchery D, Rossignol G, Poiree B, Meslier M, et al. Prevalence, risk factors and clinical implications of malnutrition in French Comprehensive Cancer Centres. Br J Cancer. 2010;102:966-71.

21. Waitzberg DL, Caiaffa WT, Correia MI. Hospital malnutrition: the Brazilian national survey (IBRANUTRI): a study of 4000 patients. Nutrition. 2001;17:573-80.

22. Wie GA, Cho YA, Kim SY, Kim SM, Bae JM, Joung H. Prevalence and risk factors of malnutrition among cancer patients according to tumor location and stage in the National Cancer Center in Korea. Nutrition. 2010;26:263-8. 